Statistical Study of Personal Computer Cluster Harmonic Currents from Experimental Measurements

Juan José Mesas, Luis Sainz, Pau Sala

Polytechnic University of Catalonia

Barcelona, Spain

juan.jose.mesas@upc.edu

sainz@ee.upc.edu

Department of Electrical Engineering

Avda. Diagonal 647, 08028 Barcelona

E.G.O. Elektro-Gerätebau GmbH

Oberderdingen, Germany

pausalaperez@gmail.com

TWS, Drives

Rote-Tor-Straße 14, 75038 Oberderdingen 


\title{
Statistical Study of Personal Computer Cluster Harmonic Currents from Experimental Measurements
}

\begin{abstract}
The net harmonic currents generated by a cluster of desktop personal computers are studied statistically. Personal computers (PCs) are single-phase non-linear loads with low individual consumption but significant collective distortion effects, as many of them can be connected to the same bus (personal computer cluster). This article reports experimental measurements of harmonic currents injected by single-phase rectifiers and seven personal computer types during four operating modes. The probability density functions (pdfs) of the harmonic currents (magnitude and phase angle) are investigated from the measurements. An analytical procedure to obtain these probability density functions for any typical personal computer working session is described and experimentally validated. The obtained probability density functions are found to be applicable to large-scale harmonic penetration statistical studies. Furthermore, attenuation and diversity effects are analyzed from the previous probability density functions, and the harmonic current cancellation effect on personal computer clusters is investigated with Monte Carlo simulations.
\end{abstract}

Keywords: personal computers; personal computer clusters; harmonic currents; statistical analysis; probability density functions; large-scale harmonic penetration; attenuation effect; diversity effect; cancellation effect; Monte Carlo simulations

\section{Introduction}

Due to the growing number of non-linear loads connected to the distribution system, harmonics are receiving increasing attention. For example, they are the topic of several international standards (e.g., [1]). Some of these loads are low-power single-phase loads in the $200-300 \mathrm{~W}$ range that use solid-state switching devices as their power supply (personal computers [PCs], televisions, etc.). Although their individual consumption is small, their collective effect can be significant because many of them are often connected to the same bus. For this reason, several studies trying to predict their harmonic current injection have been conducted in the last decades. Some are based on theoretical models and simulation performances [2-7] and evaluate the harmonic current magnitude and the phase angle to determine harmonic current content completely and study the attenuation and diversity effects on net harmonic currents of PC clusters. 
The harmonic current spectrum of PCs is characterized from simulations [7-10] or experimental measurements [7,11-14]. In these studies, such issues as impact on networks $[8-11,13]$, cancellation phenomenon $[7,11,12,14]$, and attenuation phenomenon $[7,11,12]$ are addressed. In addition to the previous characterization, other works include statistical studies describing the probability density functions (pdfs) of the fundamental and harmonic currents as obtained from simulations [15] or experimental measurements [16-19]. In some of these studies, such aspects as impact on networks [16, 18], cancellation phenomenon [16], and attenuation phenomenon [16] are slightly analyzed, and despite the studies remarking the importance of all three aspects, no protocol is provided to address them from a statistical point of view by using the previously obtained pdfs of the fundamental and harmonic currents.

The present article goes one step further, and not only does it provide an analytical study of the statistical characterization of PC harmonic currents from experimental measurements to offer insight into PC harmonic behaviour and attenuation and diversity phenomena, but also a protocol for large-scale harmonic penetration statistical studies. The aim of this is to facilitate future research on harmonic voltage characterization in the presence of these devices. A complete set of actual measurements was obtained by testing several single-phase rectifiers in the laboratory and monitoring seven PC types during four typical operation modes. Their fundamental and harmonic currents (magnitude and phase angle) were analysed, and the statistical characterization of the harmonic current pdfs was analytically studied from the experimental measurements. The applicability of these pdfs to large-scale harmonic penetration statistical studies was described. The net harmonic currents generated by PC clusters were examined, and Monte Carlo simulations were 
performed from the obtained experimental distributions to investigate the diversity phenomenon in these clusters.

\section{Desktop PCs}

\subsection{PC Model}

PCs use switching power suppliers as their power supply. These devices convert input $\mathrm{AC}$ voltage into a desired output DC voltage. Figure 1(a) shows the equivalent circuit of PC switching power suppliers for harmonic analysis [2-5]. The suppliers are composed of a single-phase uncontrolled AC/DC rectifier with an AC choke inductor $L$ (together with its associated resistance $R$ ) and a DC-smoothing capacitor $C$ that feeds a DC-to-DC block. This block converts the input DC voltage $v_{C}$ into a regulated DC voltage and is generally modelled as the resistor $R_{\mathrm{eq}}$ considering that $v_{C}$ is a low-ripple constant DC voltage thanks to a sufficiently large capacitor $C$ [2-5].

Figure 1(b) illustrates the input AC voltage $v$, the non-regulated DC voltage $v_{C}$ and the AC current $i$ measured in a rectifier with $R \approx 0.32 \Omega, L=4.9 \mathrm{mH}, C=75.8 \mu \mathrm{F}$ and $R_{\mathrm{eq}}=1200 \Omega$. The rectifier is fed with a sinusoidal voltage input of $100 \mathrm{~V}$ RMS, 0 phase angle and $50 \mathrm{~Hz}$ frequency. The measurements were made in the laboratory with a $4.5 \mathrm{kVA}$ AC ELGAR Smartwave Switching Amplifier as the power source, a YOKOGAWA DL 708 E digital scope, and a FLUKE 43B Power Quality Analyzer as the measurement devices and a laptop computer as the data processing device. The fundamental and harmonic current magnitudes and phase angles of the measured rectifier current waveform, $I_{k}$ and $\phi_{k}$ with $k=1$ to 11 , are shown in Figure 1(c). The current phase angles are referred to the input voltage angle. These fundamental and harmonic currents are basically characterized by the ratio $L \omega / R_{\mathrm{eq}}[14]$, where $\omega=2 \pi \cdot f$, and $f$ is the fundamental frequency of the input voltage $v$. 


\subsection{PC Clusters}

The equivalent single-phase circuit of PC clusters in European commercial and residential installations is shown in Figure 2. PCs and other electronic equipment are connected to the line-to-neutral low-voltage (LV) bus, thus sharing the mediumvoltage (MV)/LV transformer. The MV/LV transformer and the wall outlet circuits are represented by impedances $\underline{Z}_{\mathrm{tr}}=R_{\mathrm{tr}}+\mathrm{j} \cdot L_{\mathrm{tr}} \cdot \omega$ and $\underline{Z}_{\mathrm{b}, n}=R_{\mathrm{b}, n}+\mathrm{j} \cdot L_{\mathrm{b}, n} \cdot \omega$, respectively, where $n=1$ to $N$ and $N$ is the total number of PCs sharing the same bus. It is well known that the net harmonic currents injected by these PC clusters fed from the same point of common coupling (PCC; Figure 2), can be affected significantly by the attenuation and diversity phenomena $[2-4,7,11,16]$. The attenuation phenomenon describes the influence of non-sinusoidal voltage at the PCC bus caused by the shared system impedance on harmonic currents, especially those that are higher order $[2-4,7,11,16]$. To quantify this effect, the authors of [2] proposed the "attenuation factor of the $k$-order harmonic current":

$$
A F_{k}=\frac{I_{k}^{(\mathrm{N})}}{N \cdot I_{k}^{(1)}} \quad(k>1),
$$

where $I_{k}^{(\mathrm{N})}$ is the $k$-order net harmonic current magnitude injected by $N$ identical nonlinear loads connected in parallel to a "non-stiff" bus, and $I_{k}^{(1)}$ is the $k$-order harmonic current magnitude injected when only one of these non-linear loads is connected to the same "non-stiff" bus. This factor ranges between 0 and 1 (i.e., $0 \leq A F_{k}(\%) \leq 100$ ). Note that decreasing values result in higher attenuation levels. This phenomenon can be characterized by the short-circuit ratio, i.e., the ratio between short-circuit current at the load bus and fundamental load current $\left(R_{\mathrm{sc}}=I_{\mathrm{sc}} / I_{\mathrm{L} 1}\right.$ of [1]), or the PCC voltage crest factor, i.e., the ratio of the peak value of the PCC voltage to its RMS value $\left(K_{\mathrm{c}}=\left\{v_{\mathrm{PCC}}\right\}_{\text {peak }} /\left\{v_{\mathrm{PCC}}\right\}_{\mathrm{rms}}\right)[3,4,11,16]$. 
The diversity phenomenon describes PC harmonic current cancellation due to its different phase angles $[2,3,7,11,16]$. This can result from differences in the PC types connected at the PCC bus or in the system and load parameters of the same individual PCs (PC power level, wall outlet impedance magnitude $Z_{\mathrm{b}, n}$ or $L_{\mathrm{b}, n} \cdot \omega / R_{\mathrm{b}, n}$ ratio). To quantify the effect, the authors of [2] proposed the "diversity factor of the $k$-order harmonic current":

$$
D F_{k}=\frac{\left|\sum_{n=1}^{N} \underline{I}_{k, n}\right|}{\sum_{n=1}^{N} I_{k, n}} \quad(k>1)
$$

where $N$ is the total number of non-linear loads sharing the same bus, $\underline{I}_{k, n}$ is the $k$ order harmonic current phasor injected by the $n^{\text {th }}$ non-linear load and $I_{k, n}$ is the magnitude of this phasor. This factor ranges between 0 and 1 (i.e., $\left.0 \leq D F_{k}(\%) \leq 100\right)$. Note that decreasing values result in higher cancellation levels.

To analyze the above phenomena, three experimental tests with single-phase rectifiers (Figure 1(a)) based on the equivalent circuit of Figure 2 with a sinusoidal voltage input of a $100 \mathrm{~V}$ RMS were performed. The corresponding circuits were constructed, and the voltage waveforms were generated by an AC ELGAR Smartwave Switching Amplifier power source. The voltage and current waveforms were recorded with a YOKOGAWA DL 708 E digital scope and computer-analyzed to obtain their fundamental and harmonic components, $\underline{I}_{k}$, using the discrete Fourier transform.

- First test: One $(N=1)$ and ten $(N=10)$ identical rectifiers

$\left(R \approx 0.32 \Omega, L=4.9 \mathrm{mH}, C=75.8 \mu \mathrm{F}\right.$, and $R_{\mathrm{eq}}=1200 \Omega$; Figure 1$)$ were connected to a "non-stiff" bus with negligible wall outlet impedances $\left(R_{\mathrm{tr}} \approx 0.25 \Omega\right.$, $L_{\mathrm{tr}}=4.51 \mathrm{mH}$, and $\underline{\mathrm{Z}}_{\mathrm{b}, n} \approx 0$; Figure 2). In Figure 3(a), the currents consumed by the 
rectifier and their harmonics are compared for $N=1$ and 10, and the attenuation factors (1) are labelled for $N=10$. A significant reduction of the harmonic currents due to the attenuation phenomenon is observed. In general, attenuation can be of $50 \%$ or more, tends to increase with $N$ and is higher for high-order harmonics [2-4]. Nevertheless, as pointed out in [4], although actual voltages at the PCC are generally non-sinusoidal, it must be noted that for existing voltage distortion levels in distribution systems (approximately $2-3 \%$ ), the attenuation effect can be small enough to consider a sinusoidal PCC voltage $[6,14]$. The short-circuit ratio and PCC voltage crest factor can be used to characterize the attenuation effect, and for both cases $(N=1$ and 10$)$, they are $R_{\mathrm{sc}} \approx 451$ and 48 and $K_{\mathrm{c}}=1.39$ and 1.29 , respectively. Figure 4 shows the attenuation factor $A F_{k}$, the individual harmonic current distortion $H D_{I k}=I_{k} / I_{1}[1]$ of the single-unit rectifier, and the PCC voltage crest factor $K_{\mathrm{c}}$ as a function of the $R_{\mathrm{sc}}$ ratio for the rectifier used. Note that the results obtained in the test can be approximately located in this figure and that lower $R_{\mathrm{sc}}$ ratios result in smaller attenuation factors since the PCC voltage tends to be more flattened. A flattened voltage wave broadens the rectifier current pulse and reduces the harmonics [3, 4]; that is, more heavily loaded systems (lower $R_{s c}$ ratios) have lower $K_{\mathrm{c}}$ ratios and attenuation factors. This effect was extensively studied from the simulations in $[2,4,8]$, and from the experimental measurements in $[11,16]$.

- Second test: Two different types of rectifiers $\left(R_{1} \approx 0.32 \Omega, L_{1}=4.9 \mathrm{mH}\right.$, $C_{1}=75.8 \mu \mathrm{F}, R_{\mathrm{eq} 1}=1200 \Omega, R_{2} \approx 0.71 \Omega, L_{2}=75.8 \mathrm{mH}, C_{2}=259.8 \mu \mathrm{F}$, and $R_{\text {eq2 }}=350 \Omega$; Figure 1) were connected to a "stiff" bus with negligible wall outlet impedances $\left(\underline{Z}_{\mathrm{tr}, n} \approx 0\right.$ and $\underline{Z}_{\mathrm{b}, n} \approx 0$; Figure 2). In Figure 3(b), the rectifier consumed currents and their harmonics are compared, and the diversity factors (2) are 
labelled. A significant cancellation of the fifth harmonic current $\left(D F_{5}=46.7 \%\right)$ occurs because the fifth harmonic currents of both rectifiers $\left(I_{5,1}=0.12 \angle-12.4^{\circ} \mathrm{A}\right.$ and $\left.\underline{I}_{5,2}=0.047 \angle-179.3^{\circ} \mathrm{A}\right)$ are close to counter-phase, and therefore, their geometric sum is less than their arithmetic sum. In general, cancellation due to different PC types can be significant for low-order harmonics [3, 5].

- Third test: Two identical rectifiers $(R \approx 0.32 \Omega, L=4.9 \mathrm{mH}, C=75.8 \mu \mathrm{F}$, and $R_{\text {eq }}=1200 \Omega$; Figure 1) were connected to a "stiff" bus with non-negligible wall outlet impedances $\left(\underline{\mathrm{Z}}_{\mathrm{tr}, n} \approx 0, \underline{Z}_{\mathrm{b}, 1} \approx 0.8+j \cdot 3.2 \Omega\right.$, and $\underline{Z}_{\mathrm{b}, 2} \approx 4.2+j \cdot 36.2 \Omega$; Figure 2). In Figure 3(c), the rectifier consumed currents and their harmonics are compared and the diversity factors (2) are labelled. A small cancellation of the harmonic currents due to the diversity phenomenon is observed. In general, this effect is negligible for low-order harmonics, and cancellation occurs mainly due to different PC types rather than different system and load parameters between identical PC types sharing the PCC bus $[3,7]$.

\section{PC Experimental Measurements}

To analyze PC harmonic behaviour, the following seven PC types excluding their monitors were experimentally tested: PC1(x86 Family 6 Model 8 AT/AT Compatible), PC2 (Intel Pentium 4, 3 GHz), PC3 (Intel Pentium 4, 3.06 GHz), PC4 (Intel Pentium 4, 3.4 GHz), PC5 (Intel Pentium D, 3.06 GHz), PC6 (Intel Core 2 E4300, 1.8 MHz), and PC 7 (Intel Core 2 Quad Q8200, $2.33 \mathrm{GHz}$ ).

The following PC operating modes were analyzed with a commercial software tool that allows all major PC sub-systems to be tested for reliability and stability:

- Idle mode (Idle): User input is waited for.

- $\mathrm{CPU}$ test (CPU): Several mathematical operations are performed twice. 
- Hard disk drive access $(H D)$ : Files containing different data patterns are cyclically created, read and deleted on the local hard disk.

- $\mathrm{CD} / \mathrm{DVD}$ drive access $(C D)$ : For a data $\mathrm{CD} / \mathrm{DVD}$, the device is scanned with each file being read in turn.

The measurements were monitored with PCs supplied by a laboratory outlet and carried out with the supply network analyzer AR5-L of CIRCUTOR. For each PC type and operating mode, 60 samples of outlet voltage and $\mathrm{AC}$ current were measured to evaluate accurately the outstanding aspects of $\mathrm{PC}$ harmonic behaviour. All recorded voltage and current waveforms were stored on a hard disk and treated with customized MATLAB (The MathWorks, Natick, Massachusetts, USA) software for harmonic analysis. Thus, assuming the magnitude of the current stationary, the Fourier transform was applied to a $20-\mathrm{ms}$ rectangular window providing a $50-\mathrm{Hz}$ frequency resolution.

As an example, Figure 5 reports one of the measurements of the AC current waveforms and their RMS values (in brackets) taken during Idle mode. The outlet voltage is also plotted as a reference. Note that the currents consumed by the PCs have approximately the same shape as the rectifier current in Figure 1(b). However, their sizes (i.e. current RMS values) are not the same due the different power consumption of the PCs (Figure 6). Moreover, their peak value can be placed slightly before or after the peak value of the outlet voltage depending on the PC type. The size and peak value location influence the fundamental and harmonic current magnitudes and phase angles mainly $\left(I_{k}\right.$ and $\phi_{k}$ with $\left.k \geq 1\right)$, respectively, which are analyzed in the next section. The current waveforms of the other operating modes are similar to those of the Idle mode, mainly differing in size because of the different power consumption 
of the operating modes (Figure 6). In this way, the following comments can be made regarding Figures 5 and 6:

- As expected, the lowest power consumption occurs in Idle mode.

- The highest power consumption occurs in CPU mode. The consumption mainly depends on the processor and the frequency or clock speed (compare the consumption of PC3 and PC5, which differ in the processor, or the consumption of PC3 and PC4, which differ in the frequency). Regarding CPU frequency, it is worth mentioning that PC4 consumes the most power, whereas PC1 consumes the least.

- Consumption in HD mode depends on HD capacity and, in particular, on the spindle speed related to HD fast drive performance rather on the HD manufacturer.

- The same is true of $C D$ mode; i.e., $C D$ data capacity and read/write speed are the main factors influencing consumption.

- The consumption in both $H D$ and $C D$ modes is similar.

\section{Statistical Study of PC Harmonic Current Spectra}

To introduce the statistical analysis of PC harmonic behaviour from the laboratory measurements, Figure 7 shows the influence of the outlet voltage crest factor and PC power consumption on the harmonic currents (magnitude and phase angle) consumed by PC4 during its four operating modes. The scatter plot of the harmonic current phase angle of PC2 as a function of the crest factor and power consumption is also shown for comparison with PC4. The following must be noted from Figure 7:

- The crest factor varies only slightly during the experimental tests, and the attenuation phenomenon does not affect the harmonic currents, as confirmed from the $P$ versus $I_{k}$ plot in Figure 7, because increased power consumption does not lead to reduced harmonic current magnitudes. This is also true for the other PCs, 
the mean value of the measured crest factor being $\left\{K_{c}\right\}_{\text {mean }}=1.399$. Thus, the stochastic study of single PC fundamental and main low-order harmonic currents (magnitude and phase angle) in subsequent sections allows the statistical characterization of the time-variant harmonic behaviour of single PCs and the statistical analysis of the influence of the diversity phenomenon on harmonic current cancellation, both of them only without considering the attenuation effect.

- Regarding the diversity phenomenon, harmonic cancellation due to different PC power levels between identical PC types sharing the PCC bus is not significant because harmonic current phase angle diversity due to the PC power range is low. On the other hand, harmonic cancellation between different PC types sharing the PCC bus can be considerable because harmonic current phase angle diversity is high $\left(K_{c}\right.$ versus $\phi_{k}$ and $P$ versus $\phi_{k}$ plots for PC4 and PC2 in Figure 7).

Under these assumptions, some selected results for the seven monitored PCs and four operating modes are presented in Sections 4.1 and 4.2 to characterize harmonic current random behaviour and analyze the diversity phenomenon statistically.

\subsection{Experimental Pdfs of PC Harmonic Current Magnitudes}

As an example of PC fundamental and harmonic current magnitude variability, the black lines in Figure 8 represent the pdfs corresponding to the fundamental and main characteristic harmonic current magnitudes of PC2 recorded during the above operating modes, $p\left(I_{k} \mid\right.$ mode $)$ with mode $=$ Idle, $C P U, H D$ and $C D$. Additionally, Table 1 summarizes the pdf statistics as obtained from the PC2 fundamental and main harmonic current magnitudes.

Despite the time-variant behaviour of harmonics during operating modes, the harmonic pdf patterns are mainly the same and are governed by the fundamental pdf pattern. This is because $H D_{I k}$ variability is not significant (i.e., $H D_{I k}$ is approximately 
constant), as it can be verified from Figure 9, where the individual harmonic current distortions of PC2, $H D_{I k}$ with $k=3,5$ and 7 [1], together with their sample means and standard deviations [20], for the 60 tested samples are shown. Thus, according to the relationship between pdfs of two random variables [20], the pdfs of the harmonic current magnitudes can easily be obtained from the fundamental pdfs as follows:

$$
I_{k}=g_{k}\left(I_{1}\right) \approx \overline{H D}_{I k} I_{1} \Rightarrow p\left(I_{k} \mid \text { mode }\right)=p\left(I_{1} \mid \text { mode }\right)\left|\frac{\mathrm{d} g_{k}^{-1}}{\mathrm{~d} I_{k}}\right| \approx \frac{p\left(I_{1} \mid \text { mode }\right)}{\overline{H D}_{I k}} \quad(k>1)
$$

The gray lines in Figure 8 represent the pdfs obtained from (3) considering the $H D_{I k}$ sample means in Figure 9. It must be noted that they agree well with those obtained from measurements.

Similar results were found from the monitoring activity conducted on the other PCs. Figure 10 contains their fundamental current magnitude pdfs during the four operating modes. The sample means of the individual main harmonic current distortions, $H D_{I k}$ in [1], are also indicated in Figure 10. Thus, considering (3), the harmonic current magnitude pdfs during the four operating modes can be derived easily from the fundamental current magnitude pdfs and the sample means of the individual harmonic current distortions in this figure.

To obtain the pdfs of the PC harmonic current magnitudes, $p\left(I_{k}\right)$, during a typical PC working session, the frequency or probability of occurrence of each operating mode, $p($ mode $)$ with mode $=$ Idle, $C P U, H D$ and $C D$, must be taken into account, together with the harmonic current magnitude pdfs of each operating mode, $p\left(I_{k} \mid\right.$ mode $)$. Thus, pdfs $p\left(I_{k}\right)$ can be obtained by applying the total probability theorem [20]:

$$
p\left(I_{k}\right)=\sum_{\text {mode }} p\left(I_{k} \mid \text { mode }\right) p(\text { mode }) \quad(k \geq 1)
$$


Considering (3), (4) can be approximately rewritten as

$$
p\left(I_{k}\right) \approx \frac{1}{\overline{H D}_{I k}} \sum_{\text {mode }} p\left(I_{1} \mid \text { mode }\right) p(\text { mode }) \quad(k>1)
$$

As an example, the harmonic current magnitude pdfs $p\left(I_{k}\right)$ of the seven monitored PCs obtained from (4) (black lines) and (5) (gray lines) as well as the adopted operating mode frequency $p($ mode) are shown in Figure 11. This operating mode frequency was chosen from the authors' experience, but it could be a different one.

\subsection{Experimental Pdfs of PC Harmonic Current Phase Angles}

PC fundamental and harmonic current phase angle variability can be investigated considering all operating mode samples (i.e., $4 \times 60=240$ samples) as a single set since the operating modes have similar current phase angle variability (i.e., similar sample means and standard deviations). This can be verified from Table 1, which summarizes the pdf statistics as obtained from the PC2 fundamental and main harmonic current phase angles. For this reason and for each of the seven monitored PCs, the pdfs corresponding to the fundamental and main characteristic harmonic current phase angles, obtained during the four operating modes but analyzed as a whole set, are shown in Figure 12, $p\left(\phi_{k}\right)$. These pdfs are therefore valid for any typical PC working session. The sample means of the main harmonic current phase angles are also indicated in this figure. All current phase angles are referred to the fundamental voltage phase angle.

A small influence of the PC operating mode on PC current phase angle diversity is observed from Table 1 and Figure 12. Although no results are provided, it was verified that this effect is stronger for high-order harmonics. On the other hand, it is also observed that PC type can strongly influence PC current phase angle diversity. Thus, the diversity phenomenon due to different PC power levels (i.e., different PC 
operating modes) between identical PC types sharing the PCC bus is negligible for the main harmonics, whereas the same phenomenon due to different PC types sharing the PCC bus could become significant. Despite the fact that the pdfs of the harmonic current phase angles (Figure 12) must be considered in the study of this phenomenon, in view of the small current phase angle variation due to the PC operating mode, the sample means of PC harmonic current phase angles can be used instead of the pdfs to study approximately the cancellation effect among PC types. This substitution can also be done in any study based on these pdfs.

\section{Applicability to Large-Scale Harmonic Penetration Statistical Studies}

It is very difficult to work with exact models of non-linear loads in large-scale harmonic penetration studies. Therefore, non-linear loads are modelled as independent current sources in these studies (i.e., considering that harmonic voltages have no influence on the behaviour of non-linear loads) [21]. This facilitates their incorporation into a conventional load flow, giving rise to the modified conventional load flow [22]. Once the fundamental steady state of the network and the behaviour of non-linear loads are characterized from the modified conventional load flow, the harmonic voltages can be calculated by using the voltage nodes (buses) method as follows:

$$
\mathbf{V}_{B, k}=\mathbf{Y}_{B m, k}^{-1} \cdot \mathbf{I}_{B, k} \quad(k>1) .
$$

In (6), for a network of $n$ buses and for the $k$-order harmonic, $\mathbf{V}_{B, k}$ is a column vector with $n$ bus voltages, $\mathbf{I}_{B, k}$ is a column vector with $n$ bus injected currents obtained from the modified conventional load flow, and $\mathbf{Y}_{B m, k}$ is a square matrix with $n^{2}$ bus admittances (this matrix is known as a "modified" matrix of bus admittances because the load and generator harmonic admittances are incorporated into it). 
When considering a deterministic approach, the independent current sources that model the corresponding non-linear loads have a fixed value. Therefore, the harmonic voltages obtained from (6) are only valid for that state of load. Since it is difficult to work with exact models of non-linear loads, it is more realistic to address the problem statistically, i.e., developing probabilistic models of non-linear loads that allow not only to consider the possible variability of the state of load but also to determine the pdfs of the harmonic voltages. The present article provides simplified statistical models (pdfs of the fundamental and harmonic currents) for PCs obtained from experimental measurements.

A statistical approach to large-scale harmonic penetration studies in installations where some (or all) buses are PC clusters involves following a protocol of three steps: - Step 1. In every PC cluster, for each of the PCs and each of its operating modes, $p\left(I_{1} \mid\right.$ mode $)$ must be determined from experimental measurements.

- Step 2. In every PC cluster, for each of the PCs, $H D_{I k}$ mean values and $\phi_{k}$ mean values must be determined from experimental measurements, $p$ (mode) must be set and then $p\left(I_{k}\right)$ must be determined by using (5).

- Step 3. In the installation, Monte Carlo simulations must be performed to determine $p\left(\mathbf{V}_{B, k}\right)$ : In every PC cluster, for each Monte Carlo simulation, first the PCs' harmonic current magnitudes must be randomly varied according to their respective $p\left(I_{k}\right)$ (determined in Step 2). Second, the PCs' harmonic current phase angles adopted must be their respective mean values (determined in Step 2 as well). Third, vector $\mathbf{I}_{B, k}$ of the installation must be computed from a modified conventional load flow. Finally, vector $\mathbf{V}_{B, k}$ of the installation must be computed by using (6), and its pdf $p\left(\mathbf{V}_{B, k}\right)$ must be determined. 


\section{Statistical Study of PC Cluster Harmonic Currents}

In this section, the influence of the diversity phenomenon due to different PC types on the PC cluster harmonic spectrum is analyzed from experimental measurements and Monte Carlo simulations considering neither attenuation nor the diversity due to differences in the system and load parameters of the same individual PCs. This study can be considered as a particular case of the large-scale harmonic penetration statistical studies for which the steps to be performed were mentioned in the previous section, since the study is carried out in an installation where there is a single bus, which is a PC cluster, and the third of those steps becomes to perform Monte Carlo simulations to determine the pdfs of the diversity factors of the net harmonic currents, $p\left(D F_{k}\right)$

The currents consumed by PC1 and PC4 during Idle mode while sharing the same laboratory outlet were measured to exemplify the diversity phenomenon between both PC types. The laboratory outlet was regarded as a "stiff" bus and the PC wall outlet impedances were considered negligible. Figure 13 shows the AC current waveforms and their individual harmonic current distortions $\left(H D_{I k}\right.$ in [1]). The measured outlet voltage (also plotted as a reference) has reduced distortion $\left(H D_{V k}=0.53,1.00\right.$, and $1.01 \%$ for $k=3,5$, and 7 [1] $)$ and a crest factor close to a sinusoidal crest factor $\left(K_{c}\right.$ $=1.4)$, which does not affect the harmonic currents. It must be noted that the PC1 and PC4's fifth harmonic currents are cancelled because they can be close to counterphase (see current phase angle mean values in Figure 12). Their geometric sum is therefore less than their arithmetic sum. A smaller cancellation effect of the third and seventh harmonics is also observed. According to (2), the diversity factors of the main harmonics are $D F_{k}=81.2,24.5$, and $51.0 \%$ for $k=3,5$, and 7 , which highlights the $3^{\text {rd }}, 5^{\text {th }}$, and $7^{\text {th }}$ harmonic cancellation phenomenon. This is also evident when 
comparing the current waveforms in Figure 13, because the resulting current, $i_{\mathrm{PC} 1}+i_{\mathrm{PC} 4}$, is more sinusoidal than either of the two individual currents.

Due to the lack of actual measurements of different combinations of the studied PCs, 5000-shot Monte Carlo simulations were performed to investigate the diversity effect due to different PC types on net harmonic currents of PC clusters. Thus, based upon the experimental pdfs of PCs' harmonic currents obtained in the previous section and corresponding Monte Carlo simulations, it is possible to estimate the net harmonic currents that can be expected from a cluster of the studied PCs sharing the same "stiff" bus. For each Monte Carlo simulation, the PCs' harmonic currents (magnitudes and phase angles) were randomly varied according to the pdfs in Figures 11 and 12, the diversity factors of the net harmonic currents were computed by using (2) and their pdfs were determined. According to the type and number of PCs $\left(N_{\text {PC\# }}\right.$ with $\left.\#=1,2, \ldots, 7\right)$ considered in the study, the following scenarios were analyzed:

- Scenario 1: One PC1 and one PC4, i.e., $N_{\mathrm{PC} 1}=N_{\mathrm{PC} 4}=1$.

- Scenario 2: Different numbers of PC1 and PC4, where the number of each PC is uniformly distributed over a 1 to 5 range, i.e., $N_{\mathbf{P C} 1} \sim U(1,5)$ and $N_{\mathbf{P C} 4} \sim U(1,5)$.

- Scenario 3: Different numbers of PC1 to PC7, where the number of each PC is uniformly distributed over a 1 to 5 range, i.e., $N_{\mathbf{P C} \#} \sim U(1,5)$ with $\#=1$ to 7 . The diversity factor results are shown in black lines in Figures 14(a), 14(b), and 14(c) for Scenarios 1, 2, and 3, respectively. Note the following remarks:

- In Scenario 1 (Figure 14(a)), the harmonic cancellation effect mentioned in the previous experimental example is observed. As stated above, the $5^{\text {th }}$ harmonic cancellation is achieved because these harmonics can be close to counter-phase. Similar results are obtained for other numbers of PC1 and PC4 (e.g., 


$$
\left.N_{\mathrm{PC} 1}=N_{\mathrm{PC} 4}=2,3, \ldots\right)
$$

- In Scenario 2 (Figure 14(b)), the random variation in the number of PC1 and PC4 results in wider diversity factor pdfs than those in Scenario 1. However, the cancellation effect is similar.

- In Scenario 3 (Figure 14(c)), the cancellation effect is also observed, although it is smaller than that in Scenarios 1 and 2. This is because the dispersion in the PC harmonic current phase angles results in their difference not always being close to $180^{\circ}$, as is the case of the PC1 and PC4 cluster.

As explained in the previous section, the small variation of current phase angles due to the PC operating mode (Figure 12) allows the diversity phenomenon due to different PC types to be approximately studied using the sample means of the PC harmonic current phase angles. In this way, Monte Carlo simulations were repeated for the same scenarios but considering the current phase angle mean values in Figure 12 instead of the current phase angle pdfs. Diversity factor results are shown in gray lines in Figures 14(a), 14(b), and 14(c) for Scenarios 1, 2, and 3, respectively. It must be noted that these results agree well with those obtained considering the current phase angle pdfs (black lines). The greatest difference is in Scenario 1 but becomes smaller with increasing the number of PCs.

\section{Conclusions}

This article investigates the net harmonic currents that can be expected from a cluster of desktop PCs by using experimental measurements and Monte Carlo simulations. Although the statistical study was only conducted for a restricted number of devices, the extensive measurements provide insight into PC harmonic behaviour and attenuation and diversity phenomena in PC clusters, which can be used in 
harmonic voltage characterization studies. The main findings of the study are as follows:

- The PC experimental measurements do not allow a thorough analysis of the attenuation phenomenon. Nevertheless, the relation between the short-circuit ratio, crest factor, individual harmonic current distortion, and attenuation factor of single-phase rectifiers is examined. A plot is provided to determine approximately harmonic currents from currents consumed by rectifiers connected to a "stiff" PCC bus.

- Unlike the PC operating mode and wall outlet impedances, PC type greatly influences PC harmonic current phase angles. For this reason, only the diversity phenomenon due to the different PC types connected to the PCC bus must be considered to determine harmonic current cancellation in PC clusters.

- An analytical approach to the statistical characterization of PC harmonic currents from experimental measurements is needed. Therefore, an analytical procedure to obtain the experimental pdfs of PC harmonic currents for any typical PC working session is provided and experimentally validated. This procedure benefits from two facts:

- PC individual harmonic current distortions $H D_{I k}$ are approximately constant. Therefore, the pdfs of PC harmonic current magnitudes, $p\left(I_{k} \mid\right.$ mode $)$, can be easily approximated from the fundamental ones, $p\left(I_{1} \mid\right.$ mode $)$.

- Standard deviation of PC harmonic current phase angles during PC operating modes is small. Therefore, instead of the pdfs of PC harmonic current phase angles, $p\left(\phi_{k}\right)$, the $\phi_{k}$ mean values of PC harmonic current phase angles can be used to characterize their random behaviour.

- The previous analytical procedure leads to simplified statistical models (pdfs of the 
fundamental and harmonic currents) for PCs obtained from experimental

measurements. These models are applicable to large-scale harmonic penetration

statistical studies in installations where some (or all) buses are PC clusters. A

protocol for these studies to be carried out from the models is presented, which can

be used to determine the pdfs of the harmonic voltages (harmonic voltage

characterization studies).

- The statistical study of PC cluster harmonic currents presented in this article can be regarded as a particular case of the large-scale harmonic penetration statistical studies. Therefore, following a similar protocol, Monte Carlo simulations were performed from the experimental pdfs of the PC harmonic currents to obtain the pdfs of the diversity factors of the net harmonic currents, $p\left(D F_{k}\right)$. The study not only confirms the existence of harmonic current cancellation in PC clusters, but it also highlights its random behaviour.

\section{References}

[1] IEEE, "IEEE recommended practices and requirements for harmonic control in electrical power systems," IEEE Standard 519-1992, 1993.

[2] Mansoor, A., Grady, W. M., Chowdhury, A. H., and Samotyj, M. J., "An investigation of harmonics attenuation and diversity among distributed singlephase power electronic loads," IEEE Transactions on Power Delivery, Vol. 10, No. 1, pp. 467-473, January 1995.

[3] Mansoor, A., Grady, W. M., Staats, P. T., Thallam, R. S., Doyle, M. T., and Samotyj, M. J., "Predicting the net harmonic currents produced by large numbers of distributed single-phase computer loads," IEEE Transactions on Power Delivery, Vol. 10, No. 4, pp. 2001-2006, October 1995.

[4] Mansoor, A., Grady, W. M., Thallam, R. S., Doyle, M. T., Krein, S. D., and Samotyj, M. J., "Effect of supply voltage harmonics on the input current of single-phase diode bridge rectifier loads," IEEE Transactions on Power Delivery, Vol. 10, No. 3, pp. 1416-1422, July 1995.

[5] Carpinelli, G., Iacovone, F., Varilone, P., and Verde, P., "Single phase voltage source converters: Analytical modelling for harmonic analysis in continuous and discontinuous current conditions," International Journal of Power and Energy Systems, Vol. 23, No. 1, pp. 37-48, 2003.

[6] Mayordomo, J. G., Hernández, A., Asensi, R., Beites, L. F., and Izzeddine, M., "A unified theory of uncontrolled rectifiers, discharge lamps and arc furnaces. PART I: An analytical approach for normalized harmonic emission 
calculations," Proceedings of the 8th IEEE International Conference on Harmonics and Quality of Power (ICHQP), pp. 740-748, Athens, 14-16 October 1998.

[7] Mansoor, A., and Grady, W. M., "Analysis of compensation factors influencing the net harmonic current produced by single-phase non-linear loads," Proceedings of the 8th IEEE International Conference on Harmonics and Quality of Power (ICHQP), pp. 883-889, Athens, 14-16 October 1998.

[8] Capasso, A., Lamedica, R., and Prudenzi, A., "Estimation of net harmonic currents due to dispersed non-linear loads within residential areas," Proceedings of the 8th IEEE International Conference on Harmonics and Quality of Power (ICHQP), pp. 700-705, Athens, 14-16 October 1998.

[9] Mujović, S., Katić, V. A., and Radović, J., "Improved analytical expression for calculating total harmonic distortion of PC clusters," Electric Power Systems Research, Vol. 81, No. 7, pp. 1317-1324, July 2011.

[10] Katić, V. A., Mujović, S. V., Radulović, V. M., and Radović, J. S., "The impact of the load side parameters on PC cluster's harmonics emission," Advances in Electrical and Computer Engineering, Vol. 11, No. 1, pp. 103-110, 2011.

[11] Ahmed, E. E., Xu, W., and Zhang, G., "Analyzing systems with distributed harmonic sources including the attenuation and diversity effects," IEEE Transactions on Power Delivery, Vol. 20, No. 4, pp. 2602-2612, October 2005.

[12] Moore, P. J., and Portugués, I. E., "The influence of personal computer processing modes on line current harmonics," IEEE Transactions on Power Delivery, Vol. 18, No. 4, pp. 1363-1368, October 2003.

[13] Koval, D. O., and Carter, C., "Power quality characteristics of computer loads," IEEE Transactions on Industry Applications, Vol. 33, No. 3, pp. 613-621, May/June 1997.

[14] Sainz, L., Pedra, J., and Mesas, J. J., "Single-phase full-wave rectifier study with experimental measurements," Electric Power Systems Research, Vol. 77, No. 3-4, pp. 339-351, March 2007.

[15] Mesas, J. J., Sainz, L., and Ferrer, A., "Deterministic and stochastic study of the single-phase non-linear load behavior," Proceedings of the 8th IASTED International Conference on Power and Energy Systems (EuroPES), pp. 44-49, Corfu, 23-25 June 2008.

[16] Capasso, A., Lamedica, R., and Prudenzi, A., "Experimental characterization of personal computers harmonic impact on power quality," Computer Standards \& Interfaces, Vol. 21, No. 4, pp. 321-333, 1999.

[17] Grasselli, U., Lamedica, R., and Prudenzi, A., "Time-varying harmonics of single-phase non-linear appliances," IEEE Power Engineering Society Winter Meeting 2002, Vol. 2, pp. 1066-1071, New York, 27-31 January 2002.

[18] Lamedica, R., Sorbillo, C., and Prudenzi, A., "The continuous harmonic monitoring of single-phase electronic appliances: desktop PC and printers," Proceedings of the 9th IEEE International Conference on Harmonics and Quality of Power (ICHQP), pp. 697-702, Orlando, October 2000.

[19] Falvo, M. C., Grasselli, U., Lamedica, R., and Prudenzi, A., "Harmonics monitoring survey on office LV appliances," Proceedings of the 14th IEEE International Conference on Harmonics and Quality of Power (ICHQP), pp. 1-6, Bergamo, 26-29 September 2010.

[20] Soong, T. T., Fundamentals of Probability and Statistics for Engineers (3rd Edition), England: John Wiley \& Sons Ltd., 2005. 
[21] Korovesis, P. N., Vokas, G. A., Gonos, I. F., and Topalis, F. V., "Influence of large-scale installation of energy saving lamps on the line voltage distortion of a weak network supplied by photovoltaic station," IEEE Transactions on Power Delivery, Vol. 19, No. 4, pp. 1787-1793, October 2004.

[22] Herraiz, S., Sainz, L., and Clua, J., "Review of harmonic load flow formulations," IEEE Transactions on Power Delivery, Vol. 18, No. 3, pp. 10791087, July 2003. 
Figures

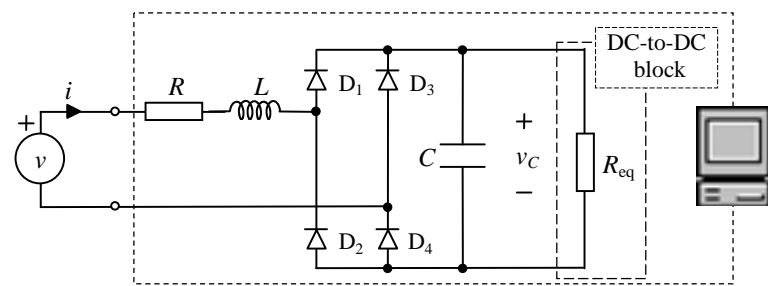

(a)

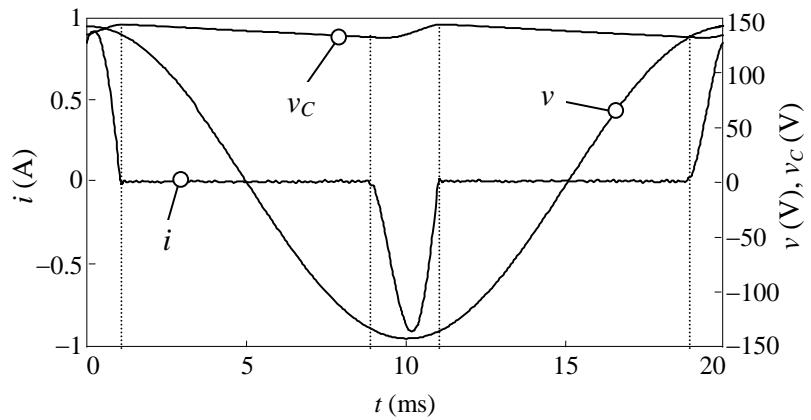

(b)
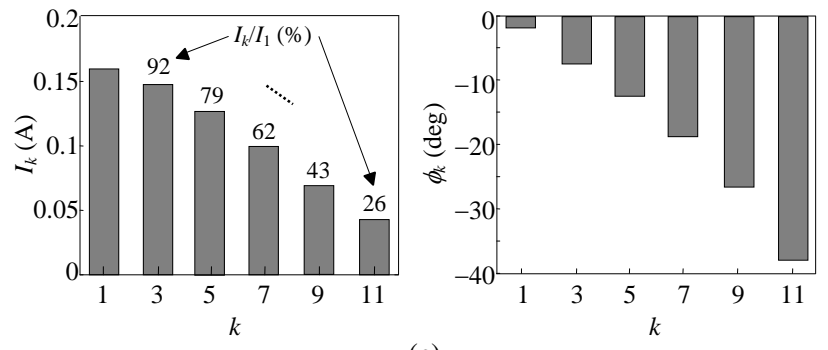

(c)

Figure 1. Single-phase uncontrolled AC/DC rectifier: (a) Equivalent circuit. (b) Measured voltage and current waveforms. (c) Harmonic current spectrum. 


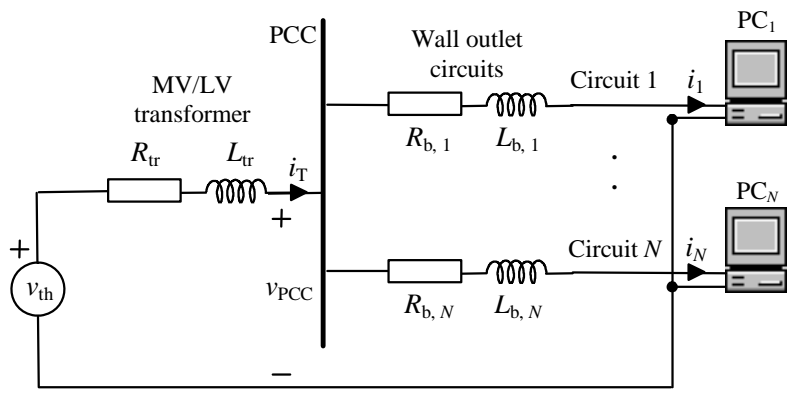

Figure 2. Equivalent single-phase circuit of PC clusters in European commercial and residential installations. 


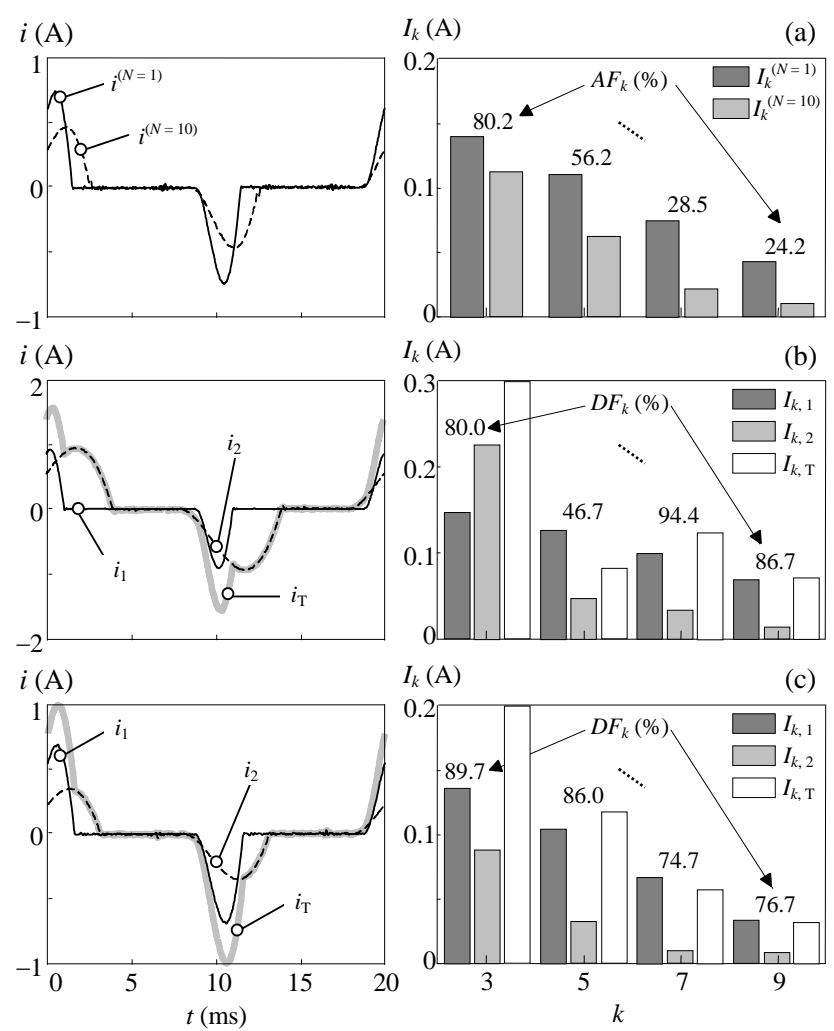

Figure 3. Rectifier currents of the laboratory tests: (a) First test. (b) Second test. (c) Third test. 


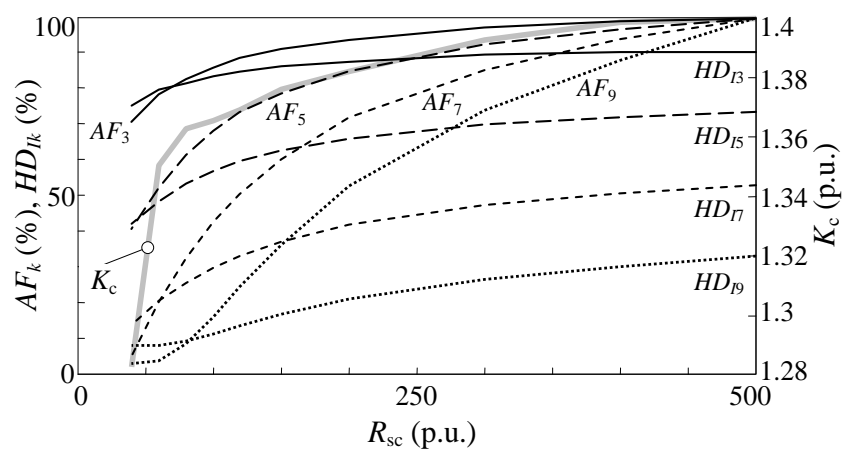

Figure 4. Short-circuit ratio influence on PCC voltage crest factor and rectifier harmonic currents. 


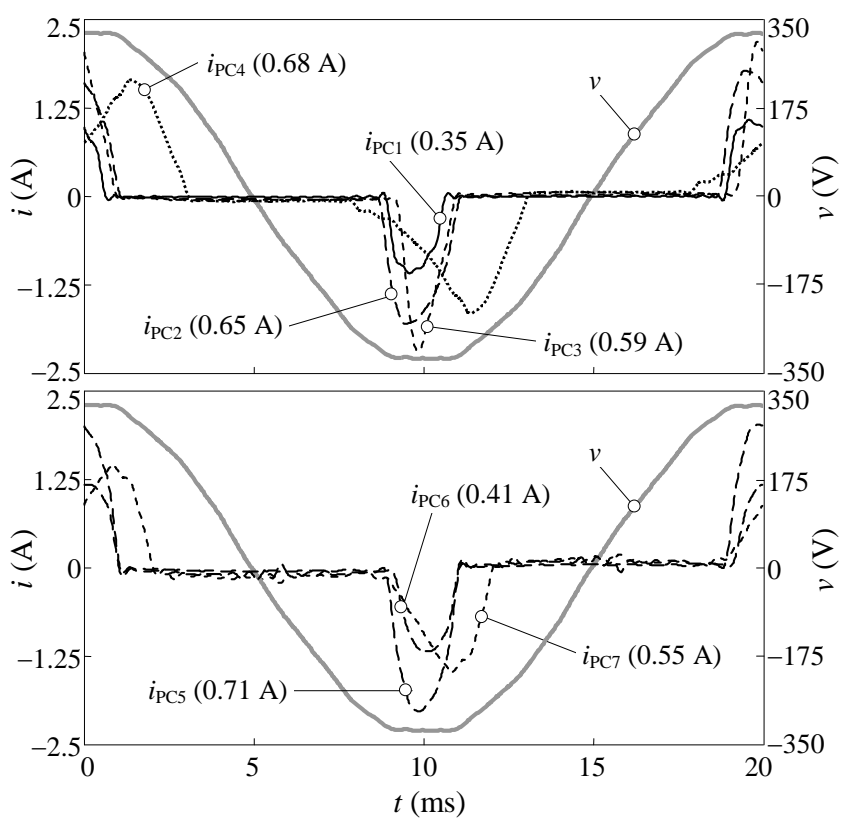

Figure 5. PC voltage and current waveforms monitored during Idle mode. 


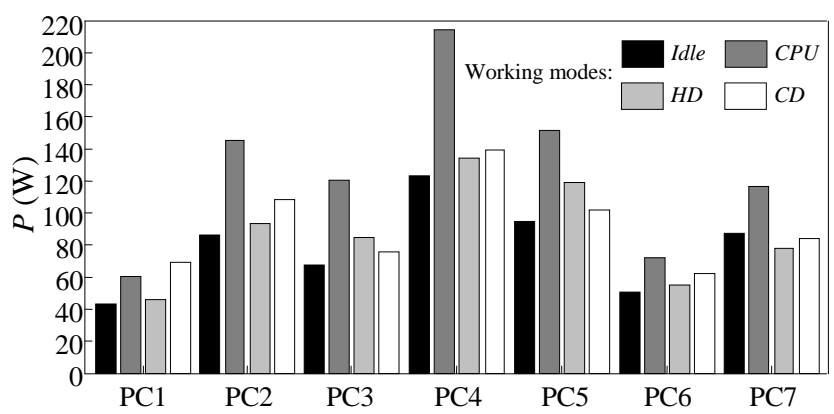

Figure 6. Mean values of PC power consumption measured for all operating modes. 


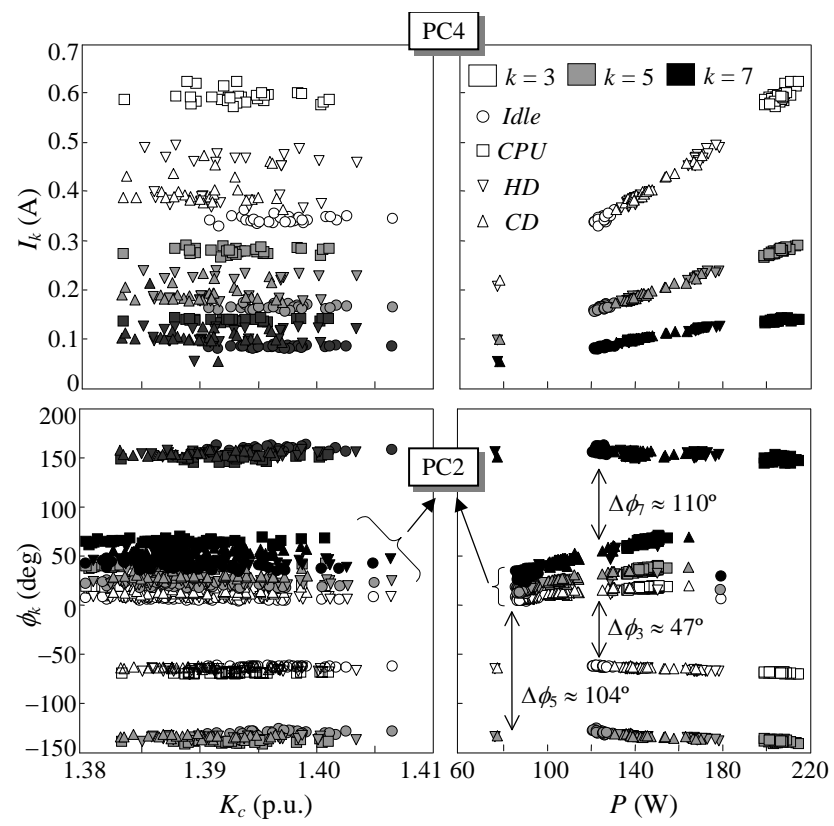

Figure 7. Influence of outlet voltage crest factor and PC power consumption on both the harmonic currents consumed by PC4 and the phase angles of the harmonic currents consumed by PC2 during their four operating modes. 

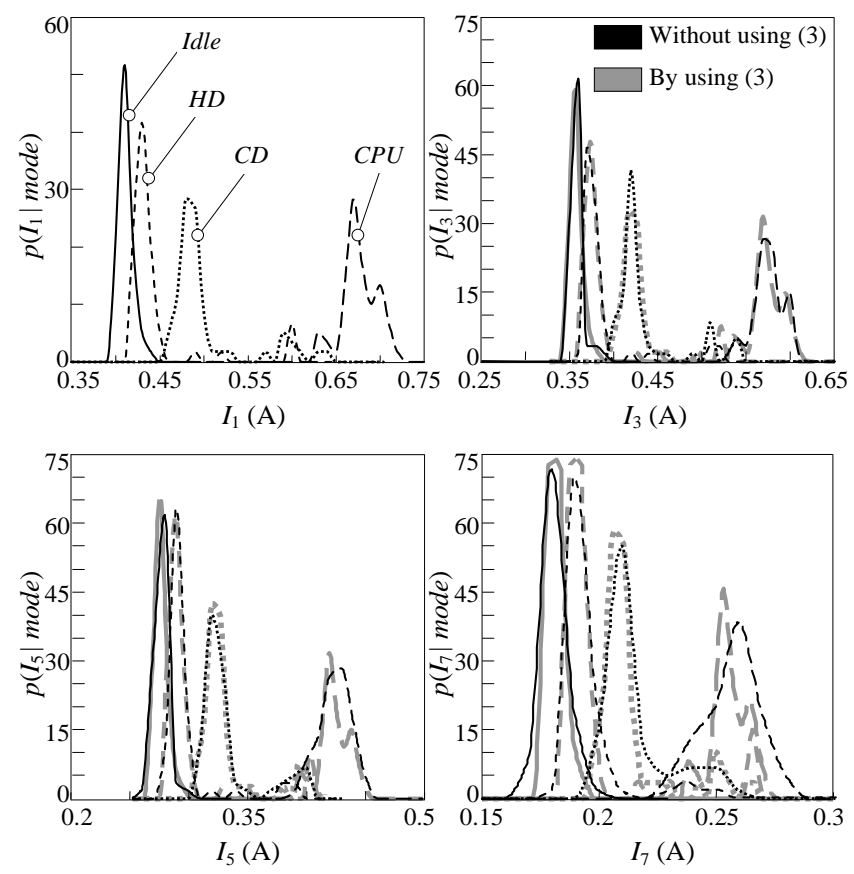

Figure 8. Pdfs of the fundamental and harmonic current magnitudes measured during the four operating modes of PC2. 


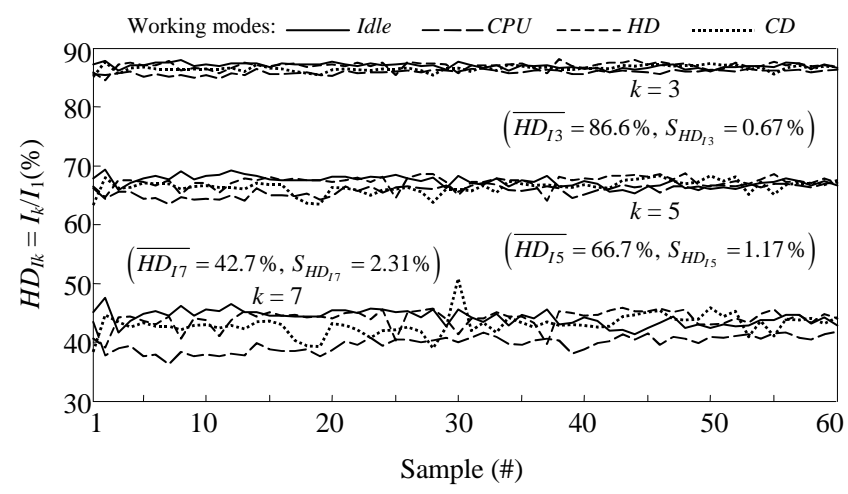

Figure 9. Individual harmonic current distortions, $H D_{I k}=I_{k} / I_{1}$, of the measured PC2 currents. 

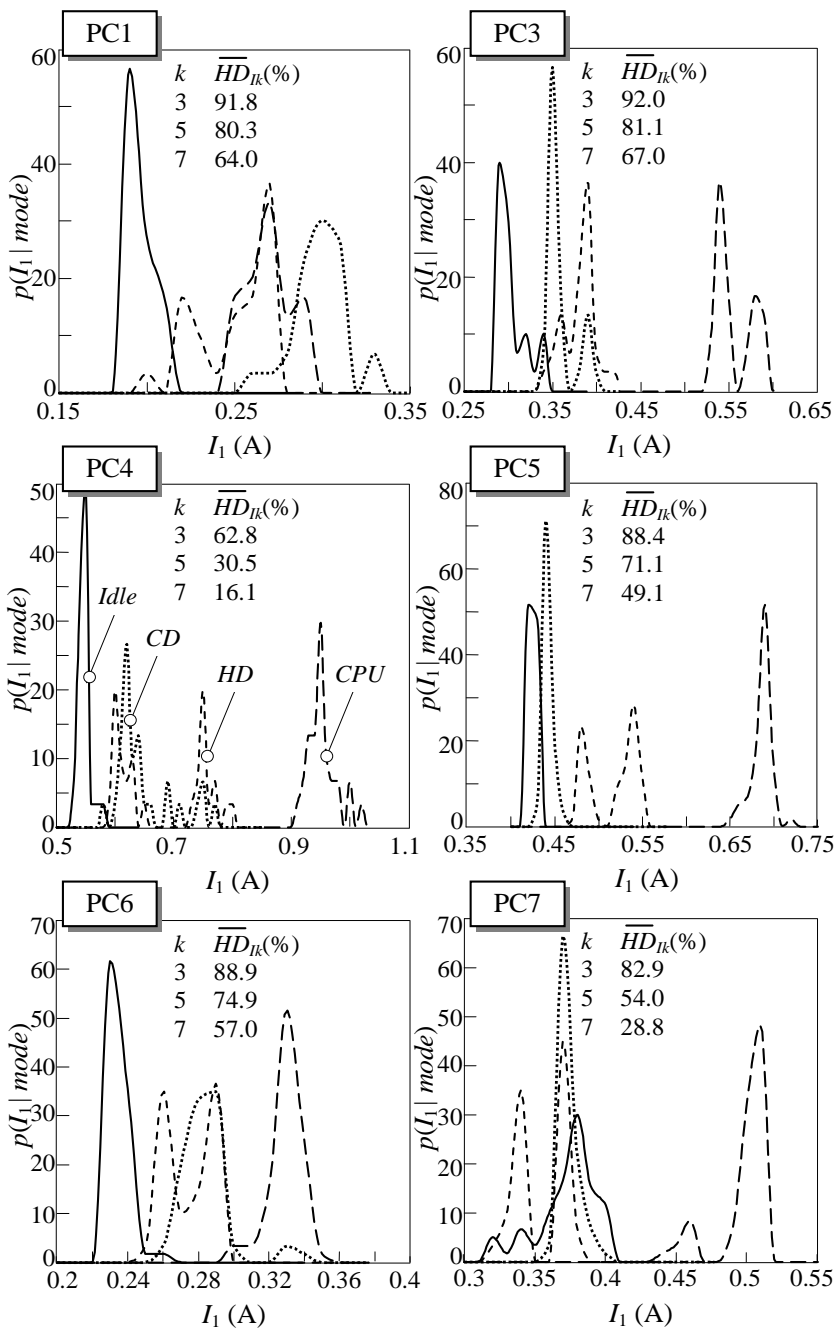

Figure 10. Pdfs of the fundamental current magnitudes of the other six PC types measured during the four operating modes. 

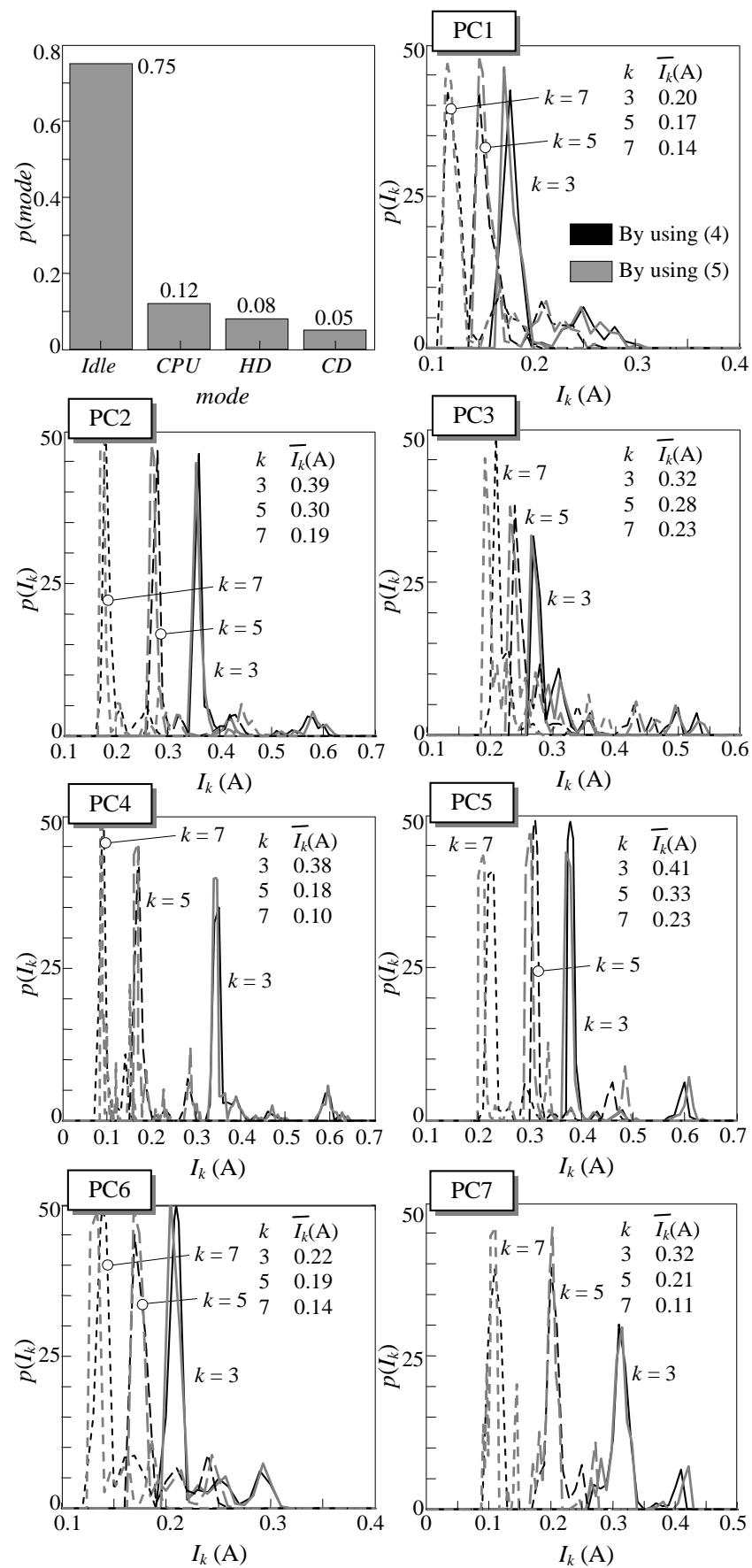

Figure 11. Experimental pdfs of the harmonic current magnitudes obtained for the seven PC types during a typical PC working session with operating mode frequencies those shown in the top left plot. 

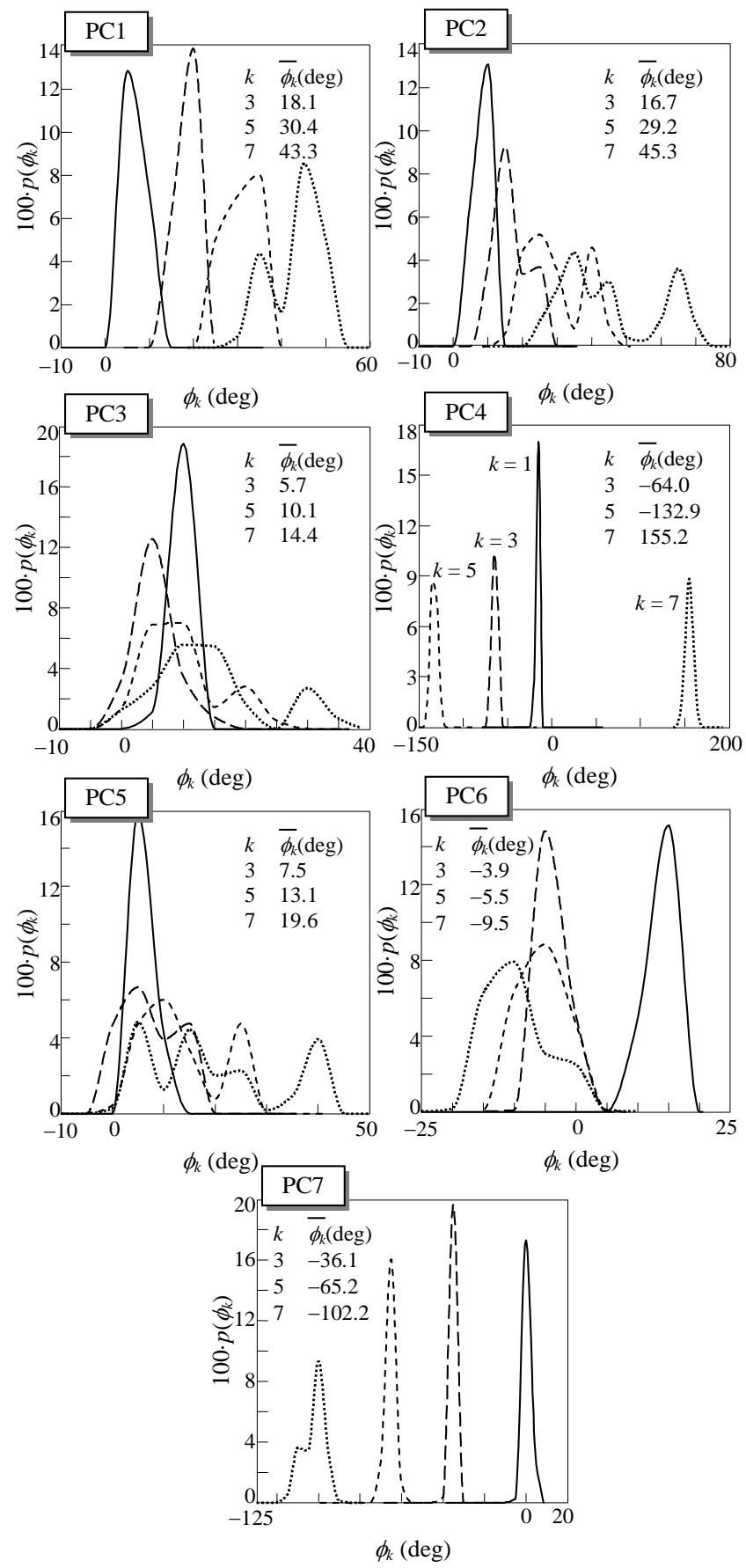

Figure 12. Experimental pdfs of the fundamental and harmonic current phase angles obtained for the seven PC types and valid for any typical PC working session. 


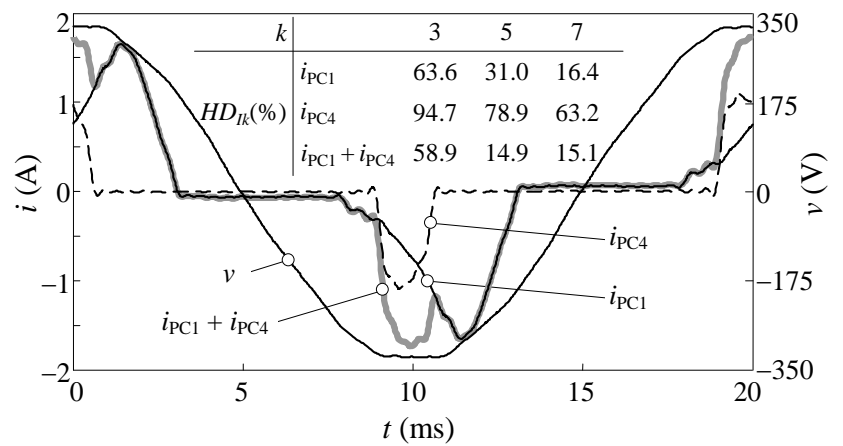

Figure 13. Voltage and current waveforms monitored for PC1 and PC4 while sharing the same outlet and operating in Idle mode. 


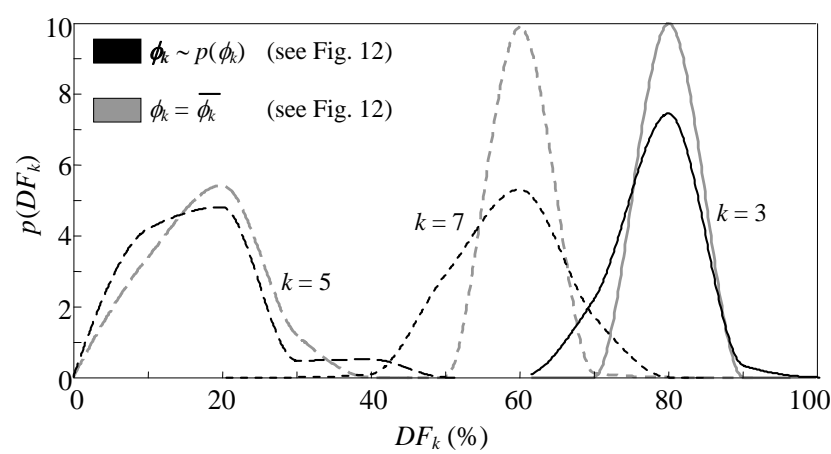

(a)

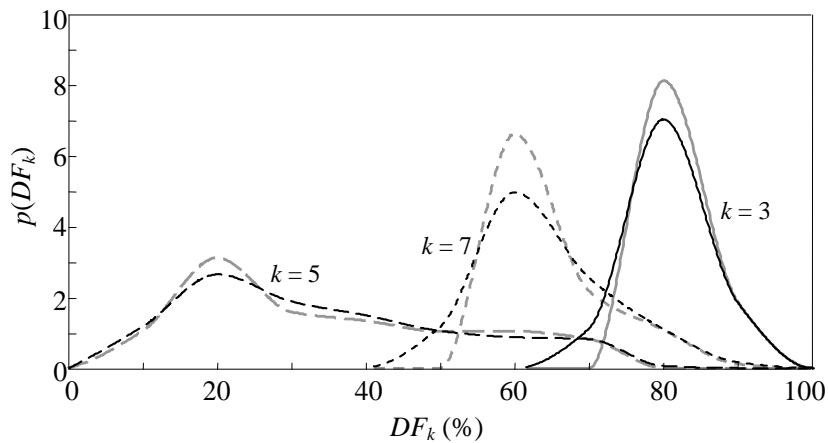

(b)

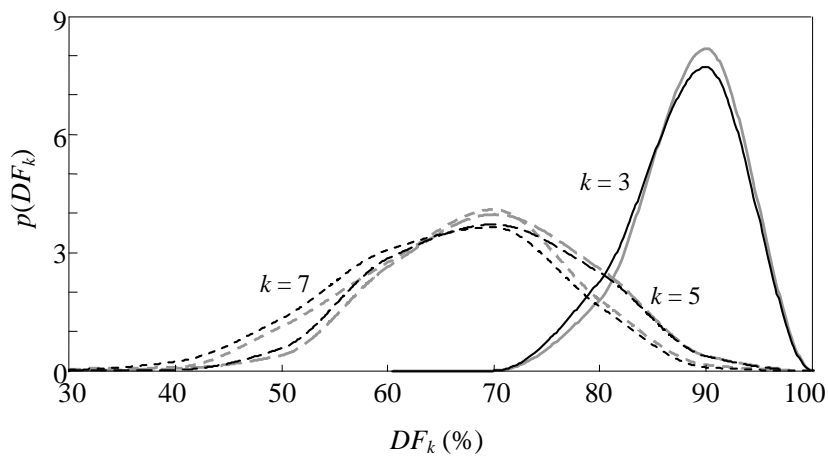

(c)

Figure 14. Diversity factor pdfs of the net $k$-th harmonic currents obtained for the three studied PC clusters: (a) Scenario 1. (b) Scenario 2. (c) Scenario 3. 
Tables

\begin{tabular}{|c|c|c|c|c|c|c|c|c|c|}
\hline & \multirow[b]{2}{*}{$k$} & \multicolumn{4}{|c|}{ Magnitude (A) } & \multicolumn{4}{|c|}{ Phase angle (deg) } \\
\hline & & Mean & Max. & Min. & $\begin{array}{l}\text { Std. } \\
\text { dev. }\end{array}$ & Mean & Max. & Min. & $\begin{array}{l}\text { Std. } \\
\text { dev. }\end{array}$ \\
\hline \multirow{4}{*}{ 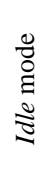 } & 1 & 0.41 & 0.44 & 0.40 & 0.008 & 7.0 & 7.7 & 6. & 0.4 \\
\hline & 3 & 0.36 & 0.39 & 0.34 & 0.007 & 12.0 & 14.2 & 9.3 & 1.3 \\
\hline & 5 & 0.28 & 0.30 & 0.26 & 0.006 & 20.6 & 24.7 & 16.1 & 2.3 \\
\hline & 7 & 0.18 & 0.20 & 0.17 & 0.005 & 31.0 & 37.8 & 23.7 & 3.7 \\
\hline \multirow{4}{*}{$\begin{array}{l}\mathbb{Z} \\
0 \\
\vdots \\
D \\
\text { Oे }\end{array}$} & 1 & 0.68 & 0.72 & 0.63 & 0.020 & 9.6 & 10.4 & 8.9 & 0.3 \\
\hline & 3 & 0.58 & 0.61 & 0.53 & 0.017 & 23.1 & 25.4 & 20.7 & 1.1 \\
\hline & 5 & 0.42 & 0.45 & 0.39 & 0.014 & 41.0 & 44.8 & 36.6 & 1.8 \\
\hline & 7 & 0.26 & 0.28 & 0.23 & 0.011 & 65.7 & 71.4 & 58.0 & 2.7 \\
\hline \multirow{4}{*}{ 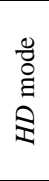 } & 1 & 0.44 & 0.61 & 0.42 & 0.045 & 7.6 & 9.3 & 6.7 & 0.6 \\
\hline & 3 & 0.39 & 0.52 & 0.36 & 0.036 & 14.3 & 21.8 & 11.5 & 2.2 \\
\hline & 5 & 0.30 & 0.39 & 0.28 & 0.025 & 24.7 & 38.5 & 20.1 & 4.0 \\
\hline & 7 & 0.20 & 0.26 & 0.18 & 0.016 & 37.5 & 61.2 & 29.7 & 6.7 \\
\hline \multirow{4}{*}{ 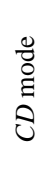 } & 1 & 0.50 & 0.64 & 0.46 & 0.045 & 8.4 & 9.7 & 7.2 & 0.5 \\
\hline & 3 & 0.44 & 0.54 & 0.40 & 0.039 & 17.5 & 23.9 & 14.3 & 2.2 \\
\hline & 5 & 0.33 & 0.40 & 0.31 & 0.027 & 30.5 & 42.6 & 25.0 & 4.0 \\
\hline & 7 & 0.21 & 0.26 & 0.20 & 0.015 & 46.8 & 68.4 & 38.3 & 6.6 \\
\hline
\end{tabular}

Table 1. Pdf statistics as obtained from the PC2 fundamental and main harmonic currents. 